

\section{MỤC LỤC}

Chính phủ điện tử, văn hóa quốc gia và tham nhũng:

Bằng chứng từ tiếp cận Bayes

Nguyễn Văn Điệp • Phạm Xuân Thu • Nguyễn Hoàng Thi

Tác động của chính sách tiền tệ đến thị trường tài chính trong bối cảnh đại dịch Covid-19: Bằng chứng thực nghiệm từ các nước ASEAN

Nguyễn Đức Trung • Lữ Hưu Chí • Trần Việt Dũng

Tác động của kiều hối đến tăng trưởng kinh tế tại các quốc gia châu Á - Vai trò của thể chế

Phạm Thanh Truyền • Hồ Thủy Tiên

Tác động của đa dạng hóa thu nhập đến ổn định tài

chính: Nghiên cứu trường hợp các ngân hàng thương mại Việt Nam

Lê Đình Luân • Nguyễn Thị Như Quỳnh • Tạ Thu Hồng Nhung

CPTPP - Cơ hội lớn cho thương mại hàng hóa của Việt Nam

- Canada trong trạng thái bình thường mới

Lê Thị Ánh Tuyết

Chất lượng dịch vụ và lòng trung thành của khách hàng trong hoạt động logistics tại Thành phố Hồ Chí Minh Hà Văn Dũng • Đặng Trương Thanh Nhàn

Sự căng thẳng trong công việc và ý định nghỉ việc của nhân viên ngân hàng trên địa bàn Thành phố Đà Nẵng Lê Thị Khánh Ly• Hồ Tấn Tuyến

Các yếu tố ảnh hưởng đến quyết định tham gia bảo hiểm y tế hộ gia đình: Nghiên cứu tại tỉnh Khánh Hòa 


\section{CONTENTS}

E-Government, National Culture and Corruption:

Evidence from Bayesian Approach

Nguyen Van Diep • Pham Xuan Thu • Nguyen Hoang Thi

The Impact of Monetary Policy on Financial Markets in The Context of The Covid-19 Pandemic: Empirical Evidence from ASEAN Countries

Nguyen Duc Trung • Lu Huu Chi • Tran Viet Dung

The Impact of Remittances on Economic Growth in Asian Countries: The Role of Institutions

Pham Thanh Truyen • Ho Thuy Tien

The Impact of Income Diversification on Financial Stability: The Case of Commercial Banks in Vietnam Le Dinh Luan • Nguyen Thi Nhu Quynh • Ta Thi Hong Nhung

CPTPP - A Great Opportunity for Trade between Vietnam and Canada in The New Normal

Le Thi Anh Tuyet

Service Quality and Loyalty of Logistics Customers in Hochiminh City

Ha Van Dung • Dang Truong Thanh Nhan

Stress in Working Life and Turnover Intention of Bankers in Da Nang City

Le Thi Khanh Ly • Ho Tan Tuyen

Factors Affecting The Decision to Participate in Household Health Insurance: A Case Study in Khanh Hoa Province Tran Thi Kim Oanh • Nguyen Viet Hong Anh 


\title{
Chính phủ điện tử, văn hóa quốc gia và tham nhũng: Bằng chứng từ tiếp cận Bayes
}

\author{
Nguyễn Văn Điệp ${ }^{(*)} \bullet$ Phạm Xuân Thu • Nguyễn Hoàng Thi
}

Ngày nhận bài: 21/5/2021 | Biên tập xong: 02/8/2021 | Duyệt đăng: 10/8/2021

\begin{abstract}
TóM TẮT: Mục tiêu của bài viết này nhằm phân tích ảnh hưởng của chính phủ điện tử (CPĐT) đến tham nhũng trong bối cảnh khác biệt văn hóa giữa các quốc gia. Phương pháp hồi quy tuyến tính theo trường phái Bayes được dùng để xử lý bộ dữ liệu bảng của 66 quốc gia trong giai đoạn 2003-2018. Kết quả nghiên cứu cho thấy ảnh hưởng mạnh mẽ và tích cực của CPĐT đến việc kiểm soát tham nhũng của các quốc gia. Bên cạnh đó, mức độ ảnh hưởng của $\mathrm{CP} \boxminus T$ đến việc kiểm soát tham nhũng tùy thuộc vào bối cảnh văn hóa quốc gia. Cụ thể, việc phát triển và tham gia CPĐT sẽ giúp kiểm soát tham nhũng hiệu quả hơn ở các quốc gia có nền văn hóa mà mức độ khoảng cách quyền lực, chủ nghĩa cá nhân, nam quyền và né tránh rủi ro cao. Ngoài ra, kết quả nghiên cứu cũng cho thấy GDP bình quân đầu người, ổn định chính trị và chính phủ hiệu quả có ảnh hưởng tích cực đến kiểm soát tham nhũng. Kết quả này hàm ý rằng, bên cạnh yếu tố văn hóa thì các yếu tố kinh tế và chính trị cũng góp phần làm giảm thiểu tham nhũng ở cấp độ quốc gia.
\end{abstract}

Từ KHÓA: Tiếp cận Bayes, tham nhũng, văn hóa, chính phủ điện tử.

Mã phân loại JEL: C11, D73, H11, Z10.

\section{Giới thiệu}

Nhiêu nghiên cứu đã phân tích các yếu tố ảnh hưởng đến tham nhũng ở cấp độ quốc gia (Nam, 2018). Trong khi đó, các yếu tố truyền thống như chính trị và kinh tế đã được xác định có tác động đến mức độ tham nhũng. Ngày nay, nhiêu quốc gia đã phát động và tham gia các chương trình chống tham nhũng như sáng kiến $\mathrm{CPĐT,} \mathrm{chính} \mathrm{phủ} \mathrm{mở} \mathrm{và} \mathrm{các} \mathrm{nỗ}$ lực minh bạch về quy trình dữ liệu, thông tin, quy định và chính sách (Park \& Kim, 2019). Trong bối cảnh đó, $\mathrm{CPĐT} \mathrm{ngày} \mathrm{càng} \mathrm{đóng} \mathrm{vai}$ trò quan trọng trong việc nâng cao hiệu quả, tính minh bạch và trách nhiệm giải trình của chính phủ. CPĐT được xem là một biểu hiện của việc ứng dụng công nghệ thông tin và truyên thông (ICT) nhằm cải thiện hiệu quả hoạt động của chính phủ và các mối quan hệ giữa công dân và chính phủ.

\footnotetext{
(*) Nguyễn Văn Điệp - Trường Đại học Mở TP.HCM; 35-37 Hồ Hảo Hớn, Phường Cô Giang, Quận 1, Thành phố Hồ Chí Minh; Email: diep.nv@ou.edu.vn.
} 
Một câu hỏi đặt ra là: "Liệu những nỗ lực bằng các sáng kiến $\mathrm{CP} Đ \mathrm{~T}$ có giúp kiểm soát tham nhũng tốt hơn so với những nỗ lực truyền thống hay không?”. Nhiều nghiên cứu thực nghiệm đã cho thấy, $\mathrm{CPĐT} \mathrm{giúp}$ kiểm soát tốt tham nhũng tại các quốc gia (Andersen, 2009; Elbahnasawy, 2014; Park \& ctg, 2019). Tuy nhiên, Nam (2018) cho rằng việc nâng cấp ICT cho các hoạt động của chính phủ có thể giúp công dân và các cơ quan chính phủ kiểm soát tham nhũng, nhưng quan điểm dựa vào ICT hoặc lấy ICT làm trung tâm lại có hạn chế trong việc đo lường tham nhũng vì tham nhũng ăn sâu vào các khía cạnh chính trị, kinh tế và văn hóa của một quốc gia riêng lẻ. Chẳng hạn, Nam (2018) và Zhao, Ahn, \& Manoharan (2017) đã cho thấy văn hóa quốc gia có ảnh hưởng đến hiệu quả của $\mathrm{CPĐT}$ trong việc giảm thiểu tham nhũng.

Trong khi các nghiên cứu trước đây sử dụng cách tiếp cận tần số để phân tích mối quan hệ giữa $\mathrm{CPĐT,} \mathrm{văn} \mathrm{hóa} \mathrm{quốc} \mathrm{gia} \mathrm{và} \mathrm{tham}$ nhũng. Nghiên cứu này sẽ sử dụng cách tiếp cận Bayes với những ưu điểm vượt trội và đáng tin cậy hơn so với cách tiếp cận tần số để phân tích ảnh hưởng của CPĐT đến tham nhũng trong bối cảnh sự khác biệt văn hóa của các quốc gia. Kết quả nghiên cứu đã bổ sung bằng chứng thực nghiệm mạnh mẽ cho thấy $\mathrm{CPĐT} \mathrm{như} \mathrm{là} \mathrm{một} \mathrm{công} \mathrm{cụ} \mathrm{chiến} \mathrm{lược}$ chống tham nhũng. Bên cạnh đó, nghiên cứu cũng cho thấy sự khác biệt văn hóa giữa các quốc gia là lý do cơ bản dẫn đến tham nhũng. Cụ thể, phát triển và tham gia CPĐT sẽ ngăn ngừa tham nhũng hiệu quả hơn ở các quốc gia có nền văn hóa mà mức độ khoảng cách quyền lực, chủ nghĩa cá nhân, nam quyền và né tránh rủi ro cao.

Phần còn lại của bài viết này được bố cục như sau: Mối quan hệ giữa $\mathrm{CPĐT,} \mathrm{văn} \mathrm{hóa}$ quốc gia và tham nhũng được trình bày trong phần cơ sở lý thuyết. Trong phần phương pháp nghiên cứu, các tác giả sẽ đi vào trình bày dũ liệu, mô hình và phương pháp nghiên cứu. Các phát hiện thực nghiệm sẽ được báo cáo trong phần kết quả và thảo luận. Phần cuối cùng, bài báo sẽ trình bày các hàm ý chính sách, hạn chế và đề xuất hướng nghiên cứu trong tương lai.

\section{Cơ sở lý thuyết và các nghiên cứu liên quan}

\subsection{Tham nhũng}

Tham nhũng và các giải pháp phòng chống tham nhũng là một trong những vấn đề được trích dẫn và thảo luận nhiều nhất trong khoa học xã hội. Định nghĩa phổ biến và được sử dụng rộng rãi về tham nhũng là "các hành vi lạm dụng chức vụ công để hưởng tư lợi trực tiếp hoặc gián tiếp" (Basyal, Poudyal, \& Seo, 2018). Tham nhũng được giải thích dựa vào mô hình người ủy nhiệm - người thừa hành khách hàng (principal - agent - client model). Mô hình này được phát triển dựa trên vấn đề bất cân xứng thông tin (Klitgaard, 1988). Trong mô hình này, những người ủy nhiệm (principal) được bâu chính thức để đại diện cho nhà nước và công dân. Mô hình giả định rằng nhũ̃ng người ủy nhiệm sử dụng các nhân viên hành chính công (người thừa hành) để cung cấp các dịch vụ công cho công dân (khách hàng). Vấn đề thông tin bất cân xứng bắt nguồn từ sự hiểu biết về hành chính công của các người thừa hành nhiêu hơn so với người được ủy nhiệm và khách hàng. Nếu các nhân viên hành chính công khai thác vấn đề thông tin bất cân xứng để lợi dụng quyền lực được giao phó và theo đuổi lợi ích của riêng mình sẽ dẫn đến tham nhũng. Theo Klitgaard (1988), mô hình này cho thấy nguồn gốc của tham nhũng bắt nguồn từ hai yếu tố: thông tin bất cân xứng và quyền lực được giao phó.

Park \& ctg (2019) cho rằng, các tài liệu vê tham nhũng chủ yếu tập trung vào ba chủ đề chính: các yếu tố quyết định tham nhũng, hậu quả của tham nhũng, và các chiến lược chống tham nhũng. Jain (2001) lập luận rằng có ba 
yếu tố quyết định tham nhũng: (i) Quyền lực tự do cân nhắc (discretionary power), bao gồm quyền lập pháp, quản lý và điều tiết; (ii) Đặc lợi kinh tế do quyền lực tạo ra; và (iii) Hình phạt và khả năng phát hiện tham nhũng thấp. Nhiều nhà nghiên cứu cũng tiến hành điêuu tra hậu quả của tham nhũng. Chẳng hạn như tham nhũng đã làm giảm đâu tư tư nhân (Seligson, 2002), giảm nguồn thu thuế của chính phủ (Nguyen \& Duong, 2022) và cuối cùng làm suy giảm tăng trưởng kinh tế (Nguyen \& Duong, 2021; Thach, Anh, \& An, 2019). Đồng thời, tham nhũng có khả năng làm bóp méo các quy trình mua sắm của chính phủ, làm gia tăng tính kém hiệu quả và không công bằng trong việc ký hợp đông với chính phủ (Rose-Ackerman, 1996). Hơn nũa, tham nhũng có xu hướng tác động tiêu cực đến nhận thức của công chúng về tính hợp pháp và hiệu quả hoạt động của chính phủ, cũng như niềm tin vào chính phủ. Điều này có khả năng làm tăng sự sẵn sàng phá vỡ các quy tắc của công chúng (Villoria, Van Ryzin, \& Lavena, 2013). Cuối cùng, các chiến lược chống tham nhũng cũng được phát triển và thực hiện ở khắp nơi trên thế giới. Các giải pháp đó được phân loại thành ba cách tiếp cận khác nhau nhưng bổ sung cho nhau như cải cách hành chính, thực thi pháp luật và trao quyền cho xã hội (Park \& ctg, 2019).

\subsection{Chính phủ điện tử}

Ban đâu, CPĐT (e-government) đề cập đến việc ứng dụng ICT trong "các hoạt động thường ngày do các tổ chức công thực hiện" (Park \& ctg, 2019; Zhao \& ctg, 2017). Các hoạt động thường ngày bao gồm việc quản lý thông tin của chính phủ và cung cấp dịch vụ cho công chúng. Mục đích của CPĐT là nâng cao hiệu quả và chất lượng các hoạt động và hệ thống cung cấp dịch vụ công của chính phủ. Nhiều nhà nghiên cứu cho rằng, $\mathrm{CP} Đ T$ có thể là một công cụ chống tham nhũng hiệu quả bằng cách: (i) Tăng khả năng tiếp cận thông tin của công chúng; (ii) Đảm bảo các quy tắc được minh bạch và áp dụng trong các quyết định cụ thể; (iii) Xây dựng khả năng theo dõi các quyết định và hành động của các công chức; và (iv) Giảm quyên lực tự do cân nhắc bằng cách tiêu chuẩn hóa việc cung cấp dịch vụ (Nam, 2018; Zhao \& ctg, 2017).

Manoharan (2013) đã tổng hợp mô hình $\mathrm{CPĐT}$ được phát triển qua ba giai đoạn: thông tin điện tử (e-information), giao dịch điện tử (e-transaction) và tham gia điện tử (e-participation). Trong mỗi giai đoạn, CPĐT có thể giảm thiểu tham nhũng bằng những cách khác nhau. Chẳng hạn, thông tin điện tử có thể làm giảm vấn đề thông tin bất cân xứng, giao dịch điện tử có thể loại bỏ một phần quyên lực tự do cân nhắc và đặc lợi kinh tế và cuối cùng, tham gia điện tử cho phép công dân giám sát hành vi của công chức và tham gia vào các quá trình như lập ngân sách có sự tham gia, điều trần trực tuyến, bỏ phiếu,...

\subsection{Văn hóa quốc gia}

Hofstede định nghĩa văn hóa quốc gia là “các chương trình tập hợp trong tiềm thức con người để phân biệt các thành viên của nhóm người này với nhóm người khác" (Hofstede, 1984). Trong khi đó, định nghĩa văn hóa của Berrell (2021) thì cụ thể hơn, theo đó "văn hóa quốc gia là các chuẩn mực, hành vi, tín ngưỡng, phong tục tập quán và các giá trị được chia sẻ bởi người dân của một quốc gia có chủ quyền (ví dụ: văn hóa dân tộc Trung Quốc hoặc Canada). Nó đề cập đến các đặc điểm cụ thể như ngôn ngữ, tôn giáo, bản sắc dân tộc và chủng tộc, lịch sử và truyên thống văn hóa".

Do sự khác biệt về văn hóa, khả năng chấp nhận hành vi tham nhũng một cách khách quan và tính dễ bị tham nhũng là khác nhau đáng kể giữa các quốc gia (Nam, 2018). Trong khi một số quốc gia coi chủ nghĩa gia đình và thân hữu là hành vi được xã hội chấp nhận, những quốc gia khác lại coi đó là hành 
vi không thể chấp nhận được về mặt văn hóa cũng như bất hợp pháp và bị trừng phạt ngay lập tức. Văn hóa cũng ảnh hưởng đến cách công chúng nhìn nhận các hoạt động của CPĐT (Zhao \& ctg, 2017).

Trong bài viết này, lý thuyết Văn hóa quốc gia của Hofstede sẽ được nhóm tác giả sử dụng vì đây là một trong những lý thuyết mạnh và được sử dụng trong nhiều nghiên cứu về văn hóa quốc gia. Lý thuyết này được thực hiện từ một cuộc khảo sát nguồn nhân lực của IBM trên toàn thế giới giai đoạn 1967-1973. Bằng cách sử dụng phương pháp phân tích nhân tố, Hofstede đã phát hiện ra sự khác biệt văn hóa tôn tại giữa các quốc gia trên thế giới (Linh, 2020; Thach, 2021). Hofstede (1984) đã phát triển một mô hình văn hóa quốc gia bao gồm nhiêu khía cạnh văn hóa. Ban đâu, văn hóa của Hofstede gồm có bốn khía cạnh, đó là: khoảng cách quyền lực, chủ nghĩa cá nhân, nam quyền và sự né tránh rủi ro. Năm 1991, khía cạnh thứ năm là định hướng dài hạn được thêm vào. Đến năm 2010, khía cạnh tự thỏa mãn được thêm vào thành khía cạnh văn hóa thứ sáu của Hofstede (Linh, 2020).

\subsection{Mối quan hệ giữa chính phủ điện tử, văn hóa quốc gia và tham nhũng}

Nhiều nhà nghiên cứu đã sử dụng phương pháp định lượng và định tính để xem xét các mối quan hệ giữa CPĐT và tham nhũng. Hầu hết các nhà nghiên cứu đã tiến hành so sánh vĩ mô định lượng giữa các quốc gia (Andersen, 2009; Basyal \& ctg, 2018; Elbahnasawy, 2014; Krishnan, Teo, \& Lim, 2013; Lio, Liu, \& Ou, 2011; Park \& ctg, 2019; Zhao \& ctg, 2017) và phần lớn kết quả nghiên cứu đều cho thấy CPĐT sẽ giúp làm giảm thiểu tham nhũng.

Andersen (2009) sử dụng dũ liệu bảng của 149 quốc gia trong giai đoạn 1996-2006 và phương pháp hồi quy OLS và 2 SLS để phân tích tác động của CPĐT đối với việc giảm tham nhũng. Tác giả kết luận rằng, $\mathrm{CPĐT}$ làm giảm tham nhũng đáng kể. Kết quả cũng tương tự khi tác giả kiểm soát được hai yếu tố dự báo tham nhũng: GDP bình quân đầu người và tự do báo chí. Sử dụng mô hình phương sai thay đồi (modeling heteroskedasticity) khi xem xét mối quan hệ giũa $\mathrm{CPĐT}$ và tham nhũng trên bộ dữ liệu bảng toàn câu của 176 quốc gia trong giai đoạn 2003-2014, Basyal \& ctg (2018) không tìm thấy bằng chứng có ý nghĩa thống kê cho thấy CPĐT có tác động tích cực đến việc giảm thiểu tham nhũng. Tuy nhiên, có bằng chứng mạnh mẽ về tác động tích cực của hiệu quả chính phủ, sự ổn định chính trị và tình trạng kinh tế của một quốc gia đến việc giảm thiểu tham nhũng. Elbahnasawy (2014) tiến hành một nghiên cứu thực nghiệm để điều tra tác động của việc áp dụng $\mathrm{CPĐT} \mathrm{và} \mathrm{internet} \mathrm{đến} \mathrm{việc}$ kiềm chế tham nhũng của 160 quốc gia trong giai đoạn 1995-2009. Kết quả phân tích bằng mô hình hiệu ứng ngẫu nhiên (REM) và mô hình moments tổng quát (GMM) trên bộ dũ liệu bảng không cân bằng cho thấy, $\mathrm{CPĐT} \mathrm{là}$ một công cụ mạnh mẽ trong việc giảm thiểu tham nhũng nhờ việc áp dụng internet nhiêu hơn (thông qua cơ sở hạ tâng viễn thông và phạm vi và chất lượng của các dịch vụ trực tuyến). Các tác động tương tác giũa $\mathrm{CPĐT}$ và việc áp dụng internet cho thấy cả hai đóng vai trò bổ sung trong các chương trình chống tham nhũng. Bên cạnh đó, kết quả kiểm định quan hệ nhân quả Granger cho thấy mối quan hệ nhân quả một chiêu từ CPĐT đến tham nhũng, trong khi đó mối quan hệ nhân quả hai chiêu giữa việc sử dụng internet và tham nhũng cũng được tìm thấy. Krishnan \& ctg (2013) xem xét vai trò và đóng góp của CPĐT đối với mức độ tham nhũng, sự thịnh vượng kinh tế và suy thoái môi trường của các quốc gia. Kết quả phân tích bằng mô hình cấu trúc tuyến tính (SEM) trên bộ dũ̃ liệu của 105 quốc gia trong giai đoạn 2004-2008 đã cho thấy mối quan hệ đáng kể giũa: (i) Sự trưởng thành của $\mathrm{CPĐT} \mathrm{và} \mathrm{tham} \mathrm{nhũng;} \mathrm{và} \mathrm{(ii)} \mathrm{Sự} \mathrm{trưởng} \mathrm{thành}$ của $\mathrm{CPĐT,} \mathrm{sự} \mathrm{thịnh} \mathrm{vượng} \mathrm{kinh} \mathrm{tế} \mathrm{và} \mathrm{suy} \mathrm{thoái}$ 
môi trường thông qua các tác động trung gian của tham nhũng. Các phát hiện cho thấy rằng, mặc dù sự trưởng thành của CPĐT không đóng góp vào sự thịnh vượng kinh tế và suy thoái môi trường, nhưng nó có thể được thực hiện một cách gián tiếp thông qua các tác động của nó đối với việc giảm thiểu tham nhũng. Lio \& ctg (2011) đã sử dụng mẫu gồm 70 quốc gia trong giai đoạn 1998-2005 để phân tích mối quan hệ của việc sử dụng internet và giảm thiểu tham nhũng. Kết quả phân tích bằng phương pháp GMM cho thấy, bằng chứng có ý nghĩa thống kê nhửng không cao của việc sử dụng internet đối với việc giảm thiểu tham nhũng. Các tác giả nhận xét rằng, việc sử dụng internet có khả năng giảm thiểu tham nhũng, nhưng tiềm năng của nó vẫn chưa được phát huy hết. Bên cạnh đó, các tác giả cũng tìm thấy rằng mối quan hệ nhân quả hai chiều giữa việc sử dụng internet và tham nhũng. Park \& ctg (2019) cũng tiến hành một phân tích thực nghiệm nhằm xem xét $\mathrm{CPĐT} \mathrm{có} \mathrm{làm} \mathrm{giảm} \mathrm{tham} \mathrm{nhũng} \mathrm{giữa} \mathrm{các}$ quốc gia hay không. Các tác giả sử dụng dũ liệu bảng của 214 quốc gia từ năm 2003 đến năm 2016 và sau đó phân tích dũ liệu bảng dựa trên mô hình hiệu ứng cố định (FEM). Kết quả phân tích cho thấy CPĐT làm giảm đáng kể tham nhũng, trong khi tác động của chính phủ mở với tư cách là một loại $\mathrm{CPĐT} \mathrm{là} \mathrm{không} \mathrm{rõ} \mathrm{ràng.}$ Tuy nhiên, ở những quốc gia có hệ thống pháp luật hiệu quả hơn, chính phủ cởi mở có nhiêu khả năng giảm tham nhũng hơn ở những nước có hệ thống pháp luật kém hiệu quả hơn (Park \& ctg, 2019).

Bên cạnh đó, mối quan hệ giữa tham nhũng và $C P \bigoplus T$ ở các quốc gia khác nhau phụ thuộc vào bối cảnh văn hóa. Một số đặc điểm văn hóa quốc gia nhất định có thể thúc đẩy các cá nhân chấp nhận hoặc từ chối tham nhũng. Ngoài ra, văn hóa quốc gia có thể ảnh hưởng đến hiệu quả mong muốn của $\mathrm{CPĐT} \mathrm{thông}$ qua việc ảnh hưởng của nó lên việc áp dụng, chấp nhận và sử dụng ICT (Nam, 2018). Các nghiên cứu thực nghiệm đã cho thấy sự khác biệt văn hóa quốc gia đã ảnh hưởng đến việc áp dụng và phổ biến CPĐT qua đó đã giúp kiểm soát tham nhũng.

Chẳng hạn Nam (2018) đã sử dụng bộ dũ liệu của các quốc gia trên thế giới năm 2016 để phân tích ảnh hưởng của CPĐT đến mức độ kiểm soát tham nhũng. Bên cạnh đó, tác giả cũng xem xét ảnh hưởng của CPĐT đối với việc kiểm soát tham nhũng khi xem xét khác biệt về văn hóa giữa các quốc gia. Trong nghiên cứu này, nhóm tác giả sử dụng bốn khía cạnh văn hóa của Hofstede. Kết quả phân tích bằng SEM đã cho thấy sự trưởng thành của dịch vụ CPĐT góp phân kiểm soát tham nhũng và văn hóa quốc gia có vai trò điều chỉnh tác dụng chống tham nhũng của CPĐT. Cụ thể, các quốc gia có khoảng cách quyền lực càng lớn và né tránh rủi ro càng cao đã làm giảm tác dụng chống tham nhũng của CPĐT. Một nghiên cứu được thực hiện bởi Zhao \& ctg (2017) trên bộ dũ liệu của 57 quốc gia trong giai đoạn 2003-2014 cũng nhằm mục tiêu tương tự nhử nghiên cứu của Nam (2018). Tuy nhiên, Zhao \& ctg (2017) sử dụng năm khía cạnh văn hóa của House \& ctg (2004). Kết quả hôi quy bằng FEM đã cho thấy, CPĐT đã giúp kiểm soát tốt tham nhũng của các quốc gia. Đông thời, kết quả nghiên cứu cũng cho thấy sự phát triển của $\mathrm{CPĐT} \mathrm{ảnh}$ hưởng đến tham nhũng nhiều hơn ở các quốc gia có nền văn hóa có mức độ né tránh rủi ro thấp và khoảng cách quyền lực thấp.

\section{Phương pháp nghiên cứu}

\subsection{Mô hình và giả thuyết nghiên cứu}

Như đã trình bày ở phân trên, các nghiên cứu trước đã cho thấy sự phát triển của CPĐT đã giúp kiểm soát tốt tham nhũng tại các quốc gia (Andersen, 2009; Elbahnasawy, 2014; Krishnan \& ctg, 2013; Lio \& ctg, 2011; Park \& ctg, 2019). Điều này cho thấy sự phát triển CPĐT đầy thúc đầy tính minh bạch bằng cách tăng khả năng tiếp cận của chính phủ. Tính minh bạch được tăng lên nhờ quyền truy cập 
trực tuyến vào các giao dịch, dịch vụ, tài liệu và cơ sở dữ liệu ở mọi lúc, mọi nơi khiến các quan chức chính phủ khó có hành vi tham nhũng (Nam, 2018). Hơn nữa, Jaeger (2006) cho rằng, sự phát triển của $\mathrm{CPĐT} \mathrm{giúp} \mathrm{người}$ dân dễ dàng thể hiện mối quan tâm của họ và cung cấp thông tin đâu vào của người dân. Tính minh bạch được nâng cao bởi sự phát triển của $\mathrm{CP} T$ cho thấy tiềm năng của $\mathrm{CPĐT}$ như là công cụ chống tham nhũng của các quốc gia. Dựa trên thảo luận này, giả thuyết nghiên cứu thứ nhất được phát biểu như sau:

$H_{1}$ : Sự phát triên của CPĐT có ảnh hưởng tích cực đến việc kiểm soát tham nhũng của các quốc gia.

Zhao \& ctg (2017) và Nam (2018) đã cho thấy, sự khác biệt văn hóa có ảnh hưởng đến việc áp dụng và phổ biến CPĐT qua đó đã giúp kiểm soát tham nhũng. Cụ thể, văn hóa quốc gia ảnh hưởng đến hiệu quả chống tham nhũng của CPĐT theo hai cách, đó là: (i) Mức độ dễ xảy ra tham nhũng trong nền văn hóa; và (ii) Mức độ thân thiện với công nghệ trong nền văn hóa. Trên cơ sở này, giả thuyết nghiên cứu thứ hai được phát biểu như sau:

$H_{2}$ : Các khía canh văn hóa quốc gia đóng vai trò điêu tiết trong mối quan hệ giũa CPĐT và kiểm soát tham nhũng của các quốc gia.

Dựa trên hai giả thuyết này, nhóm tác giả xây dựng mô hình nghiên cứu có dạng:

$$
\mathrm{CPI}=\alpha_{0}+\beta_{\mathrm{i}} \mathrm{EGOV}+\gamma_{\mathrm{i}} \mathrm{Z}+\varepsilon .
$$

Trên cơ sở Phương trình 1, Phương trình 2 sẽ được bổ sung thêm biến tương tác giữa CPĐT và văn hóa quốc gia như sau:

$$
\begin{array}{r}
\mathrm{CPI}=\alpha_{0}+\beta_{\mathrm{i}} \mathrm{EGOV}+\gamma_{\mathrm{i}} \mathrm{Z}+\delta_{\mathrm{i}} \mathrm{EGOVx} \mathrm{CN} \\
+\varepsilon,
\end{array}
$$

Trong đó: CPI - chỉ số cảm nhận tham nhũng; EGOV - những chỉ số đại diện cho CPĐT; Z - tập hợp các biến kiểm soát, bao gồm GDP bình quân đầu người, $\mathrm{PS}$ - ổn định chính trị, $\mathrm{GE}$ - chính phủ hiệu quả; $\mathrm{CN}$ - văn hóa quốc gia theo Hofstede; và $\varepsilon$ - sai số của mô hình hồi quy.

\subsection{Dũ liệu}

Bảng 1 cung cấp tên biến và nguồn thu thập dữ liệu. Trong đó, biến phụ thuộc là chỉ số cảm nhận tham nhũng $(\mathrm{CPI})$, chỉ số này đo lường mức độ tham nhũng của từng quốc gia trên thang điểm từ 0 đến 10 và từ 0 đến 100 (từ năm 2012 đến nay). Các quốc gia có điểm số CPI càng cao cho thấy mức độ tham nhũng càng thấp.

Biến độc lập chính bao gồm $\mathrm{CPĐT}$ $(\mathrm{EGOV})$ và văn hóa quốc gia $(\mathrm{CN})$. Để đo lường việc thực hiện $\mathrm{CPĐT,} \mathrm{thứ} \mathrm{nhất} \mathrm{nhóm}$ tác giả sử dụng chỉ số phát triển $\mathrm{CPĐT}$ (EGDI). Chỉ số này đo lường mức độ sẵn sàng và năng lực của các chính phủ trong việc ứng dụng ICT để cung cấp các dịch vụ công. EGDI được đo lường trên thang điểm

\begin{tabular}{|c|c|c|}
\hline Ký hiệu & Tên biến & Nguồn dữ liệu \\
\hline \multicolumn{3}{|c|}{ Biến phụ thuộc } \\
\hline CPI & Chỉ số cảm nhận tham nhũng & Tổ chức Minh bạch Quốc tế (TI) \\
\hline \multicolumn{3}{|c|}{ Biến độc lập } \\
\hline \multicolumn{3}{|c|}{ - CPĐT (GOV) } \\
\hline EGDI & Chỉ số phát triển CPĐT & $\begin{array}{l}\text { Ủy ban các vấn đề kinh tế- xã hội của Liên Hợp quốc } \\
\text { (UNDESA) }\end{array}$ \\
\hline EPI & Chỉ số tham gia CPĐT & $\begin{array}{l}\text { Ủy ban các vấn đề kinh tế-xã hội của Liên Hợp quốc } \\
\text { (UNDESA) }\end{array}$ \\
\hline
\end{tabular}
từ 0 đến 1 (các quốc gia có điểm số càng cao

Bảng 1: Ký hiệu biến và nguồn dữ liệu 
Bảng 1: Ký hiệu biến và nguồn dữ liệu (tiếp theo)

\begin{tabular}{|c|c|c|}
\hline \multicolumn{1}{|c|}{ Ký hiệu } & Tên biến \\
\hline Biến độc lập \\
\hline • Văn hóa quốc gia (CN) \\
\hline PDI & Khoảng cách quyền lực & Nguồn dữ liệu \\
\hline IDV & Chủ nghĩa cá nhân & geerthofstede.com \\
\hline MAS & Nam quyền & geerthofstede.com \\
\hline UAI & Né tránh rủi ro & geerthofstede.com \\
\hline • Biến kiểm soát & geerthofstede.com \\
\hline GDP & $\begin{array}{c}\text { GDP bình quân đầu người theo } \\
\text { giá so sánh năm 2010 (USD) }\end{array}$ & Chỉ số Phát triển Thế giới (WDI) \\
\hline PS & Ổn định chính trị & Chỉ số Phát triển Thế giới (WDI) \\
\hline GE & Chính phủ hiệu quả & Chỉ số Phát triển Thế giới (WDI) \\
\hline
\end{tabular}

Nguồn: Tổng hợp của nhóm tác giả.

cho thấy có sự sẵn sàng và khả năng cao trong việc ứng dụng ICT để cung cấp các dịch vụ công) dựa trên trung bình có trọng số của ba chỉ số thành phần của CPĐT, đó là: (i) Chỉ số dịch vụ công trực tuyến (OSI); (ii) Chỉ số hạ tầng viễn thông (TII); và (iii) Chỉ số nguồn nhân lực (HCI). Bên cạnh đó, nhóm tác giả sử dụng thêm chỉ số tham gia CPĐT (EPI) để đại diện cho EGOV. Chỉ số này cung cấp thông tin chi tiết về cách các quốc gia sử dụng các công cụ trực tuyến để trao quyền cho người dân và thúc đẩy sự tham gia của họ. Tham gia CPĐT mở rộng các khía cạnh của EGDI bằng cách phân tích việc sử dụng các dịch vụ trực tuyến của chính phủ để chia sẻ thông tin với người dân và thu hút họ tham gia vào việc cân nhắc chính sách và quá trình ra quyết định. Nó cũng được đánh giá trên thang điểm từ 0 đến 1 , với điểm số cao hơn cho thấy sự tương tác nhiều hơn giữa chính phủ và người dân và mức độ tham gia cao hơn của công dân vào quá trình ra quyết định.

Trong nghiên cứu này, nhóm tác giả sử dụng các khía cạnh văn hóa quốc gia theo Hofstede để đại diện cho văn hóa quốc gia. Trên cơ sở nghiên cứu của Nam (2018), nhóm tác giả sử dụng bốn khía cạnh, đó là Khoảng cách quyền lực (PDI), Chủ nghĩa cá nhân (IDV), Nam quyền (MAS) và Né tránh rủi ro (UAI) để đại diện cho sự khác biệt văn hóa giữa các quốc gia.

Các biến kiểm soát bao gồm GDP (GDP bình quân đầu người theo giá so sánh năm 2010), PS (Ổn định chính trị) và GE (Chính phủ hiệu quả) được đưa vào mô hình nghiên trên cơ sở các nghiên cứu trước (Basyal \& ctg, 2018; Elbahnasawy, 2014; Krishnan \& ctg, 2013; Zhao \& ctg, 2017).

Bài viết sử dụng bộ dũ liệu bảng của 66 quốc gia trong giai đoạn 2003-2018. Trong giai đoạn 2003-2005, EGDI được UNDESA cung cấp hàng năm, từ năm 2008 thì EGDI được cung cấp hai năm một lần $(2008,2010$, $2012,2014,2016$ và 2018). Trên cơ sở này, dũ liệu của các biến còn lại cũng được thu thập phù hợp với khung thời gian của EGDI. Trong khi đó, dữ liệu về văn hóa quốc gia được lấy từ phiên bản năm 2015 của Hofstede.

\subsection{Phương pháp nghiên cứu}

Các nghiên cứu trước đây được thực hiện theo trường phái tần số. Tuy nhiên, cách tiếp cận tần số dựa vào trị số $\mathrm{p}$ ( $\mathrm{p}$-value) bị phê phán là không đáng tin cậy (Oanh \& ctg, 2022; 
Thach \& ctg, 2019; Trafimow \& ctg, 2018; Wasserstein, Schirm, \& Lazar, 2019; Vladik \& ctg, 2019). Do đó, cần phải chuyển từ sự phụ thuộc vào trị số $\mathrm{p}$ sang các phương pháp đáng tin cậy hơn.

Trong bài viết này, nhóm tác giả tiếp cận theo trường phái Bayes. Trường phái Bayes có nhiều ưu điểm và đáng tin cậy hơn trường phái tân số. Cụ thể, bài viết sử dụng phương pháp hồi quy tuyến tính Bayes để phân tích ảnh hưởng của $\mathrm{CPĐT} \mathrm{đến} \mathrm{việc} \mathrm{kiểm} \mathrm{soát}$ tham nhũng của các quốc gia trong bối cảnh sự khác biệt văn hóa giữa các quốc gia. Khác với trường phái tần số, mô hình Bayes giả định tất cả các tham số là ngẫu nhiên (Kruschke, Aguinis, \& Joo, 2014; Roberts \& Rosenthal, 2001). Suy luận Bayes được thực hiện dựa trên quy luật Bayes có dạng như sau:

$$
p(\theta \mid D)=p(D \mid \theta) p(\theta) / p(D)
$$

Trong đó: D - Dũ liệu quan sát được; $\theta$ - Véc tơ các tham số trong mô hình; $\mathrm{p}(\theta \mid \mathrm{D})$ - Xác suất hậu nghiệm; $\mathrm{p}(\theta)$ - Xác suất tiên nghiệm; $\mathrm{p}(\mathrm{D} \mid \theta)$ - Xác suất hợp lý.

Vì kích thước mẫu trong nghiên cứu này đủ lớn (594 quan sát) nên thông tin tiên nghiệm sẽ ít ảnh hưởng đến mức độ chính xác (accuracy) của mô hình (Oanh \& ctg, 2022). Do đó, nhóm tác giả sẽ sử dụng tiên nghiệm chuẩn cho các tham số hồi quy và tiên nghiệm inverse-gamma cho phương sai trong mô hình (Nguyen \& Duong, 2021). Phân phối hậu nghiệm sẽ được áp dụng bằng phương pháp Markov chain Monte Carlo (MCMC) thông qua thuật toán lấy mẫu Gibbs (Brooks \& ctg, 1998; Kruschke \& ctg, 2014; Thach, 2021; Ha, 2020). Điều kiện để suy luận Bayes vững là MCMC phải hội tụ và thuật toán lấy mẫu phải hiệu quả (Brooks \& ctg, 1998; Roberts \& ctg, 2001). Trong bài viết này, nhóm tác giả kiểm định sự hội tụ thông qua giá trị thống kê Rc của Gelman-Rubin. Nếu giá trị của thống kê Rc của các tham số nhỏ hơn 1,2 thì cho thấy sự hội tụ của các MCMC. Brooks \& Gelman (1998) cho rằng, thống kê Rc cung cấp sự chẩn đoán hội tụ tốt hơn so với chẩn đoán bằng biểu đồ (plots) và nó thường được dùng cho các thuật toán MCMC đa chuỗi (multi-chain $\mathrm{MCMC}$ ). Đồng thời, kích thước mẫu hiệu quả (effective sample size - ESS) được sử dụng để xác định hiệu quả lấy mẫu của thuật toán MCMC. Nếu chỉ số hiệu quả (efficiency) của các tham số đều lớn hơn 0,01 thì thuật toán MCMC đạt hiệu quả lấy mẫu (Roberts \& ctg, 2001).

\section{Kết quả và thảo luận}

\section{1. Ảnh hưởng của chính phủ điện tử đến tham nhũng}

Bảng 2 trình bày kết quả mô phỏng hậu nghiệm của Phương trình 1 bằng phương pháp hồi quy tuyến tính Bayes. Cụ thể, Bảng 2 đã so sánh giá trị trung bình của các tham số mô hình thực nằm trong khoảng mật độ hậu nghiệm 95\% của phân phối hậu nghiệm. Bảng 2 cũng cung cấp thống kê Rc của tất cả các tham số trong mô hình. Kết quả cho thấy những thống kê Rc đều nhỏ hơn 1,2 đối với tất cả các tham số của mô hình (thống kê Rc lớn nhất là 1,0002). Vì vậy, MCMC đã đạt đến sự hội tụ. Bên cạnh đó, chỉ số hiệu quả lấy mẫu của các tham số đều lớn hơn 0,01 nên thuật toán MCMC đạt hiệu quả lấy mẫu (chỉ số hiệu quả lấy mẫu nhỏ nhất lần lượt là 0,4630 và 0,4751). Thông qua kiểm định chẩn đoán hội tụ và hiệu quả lấy mẫu, kết quả cho thấy suy luận Bayes là vững.

Kết quả mô phỏng hậu nghiệm cho thấy tham số giá trị trung bình của hai biến đại điện cho chính phủ điện (EGDI và EPI) lần lượt là 5,7190 và 5,0544 . Đồng thời, xác suất ảnh hưởng dương của hai biến này đến biến kiểm soát tham nhũng (CPI) đêu là $100 \%$. Điều này cho thấy có bằng chứng rất mạnh về tác động tích cực của $\mathrm{CPĐT} \mathrm{đến} \mathrm{việc} \mathrm{kiểm}$ soát tham nhũng của các quốc gia. Vì vậy, 
Bảng 2: Kết quả mô phỏng hậu nghiệm cho Phương trình 1

\begin{tabular}{|c|c|c|c|c|}
\hline Tham số & $\begin{array}{l}\text { Giá trị } \\
\text { trung bình }\end{array}$ & $\begin{array}{l}\text { Xác suất } \\
\text { hậu nghiệm }\end{array}$ & $\begin{array}{c}\text { Giá trị } \\
\text { trung bình }\end{array}$ & $\begin{array}{c}\text { Xác suất } \\
\text { hậu nghiệm }\end{array}$ \\
\hline EGDI & $\begin{array}{c}5,7190 \\
{[3,7404 ; 7,6813]}\end{array}$ & $1,0000^{*}$ & - & - \\
\hline EPI & - & - & $\begin{array}{c}5,0544 \\
{[3,1045 ; 7,0039]}\end{array}$ & $1,0000 *$ \\
\hline GDP & $\begin{array}{c}0,0018 \\
{[0,0017 ; 0,0019]}\end{array}$ & $1,0000 *$ & $\begin{array}{c}0,0019 \\
{[0,0017 ; 0,0020]}\end{array}$ & $1,0000 *$ \\
\hline PS & $\begin{array}{c}1,5394 \\
{[-0,1354 ; 3,1831]}\end{array}$ & $0,9647^{*}$ & $\begin{array}{c}1,4931 \\
{[-0,1496 ; 3,1442]}\end{array}$ & $0,9623 *$ \\
\hline GE & $\begin{array}{c}5,9202 \\
{[3,9710 ; 7,8435]}\end{array}$ & $1,0000 *$ & $\begin{array}{c}5,8030 \\
{[3,8713 ; 7,7217]}\end{array}$ & $1,0000 *$ \\
\hline _cons & $\begin{array}{c}8,9915 \\
{[7,0611 ; 10,8905]}\end{array}$ & $1,0000 *$ & $\begin{array}{c}9,1844 \\
{[7,2363 ; 11,0877]}\end{array}$ & $1,0000 *$ \\
\hline var & $\begin{array}{c}702,0484 \\
{[607,2978 ;} \\
810,4543]\end{array}$ & - & $\begin{array}{c}716,0408 \\
{[618,8750} \\
825,4224]\end{array}$ & - \\
\hline Thống kê Rc lớn nhất & \multicolumn{2}{|c|}{1,0002} & \multicolumn{2}{|c|}{1,0002} \\
\hline $\begin{array}{l}\text { Chỉ số hiệu quả } \\
\text { lấy mẫu nhỏ nhất }\end{array}$ & \multicolumn{2}{|c|}{0,4630} & \multicolumn{2}{|c|}{0,4751} \\
\hline
\end{tabular}

* Xác suất hậu nghiệm > 0 là xác suất mà giá trị trung bình của các tham số có ảnh hưởng tích cực đến CPl; trong dấu ngoặc vuông [ ] là khoảng mật độ hậu nghiệm Nguồn: Tính toán của nhóm tác giả.

Giả thuyết 1 được chấp nhận. Kết quả này hàm ý rằng việc phát triển $\mathrm{CPĐT}(\mathrm{EGDI})$ và tham gia $\mathrm{CPĐT} \mathrm{(EPI)} \mathrm{làm} \mathrm{giảm} \mathrm{hành} \mathrm{vi} \mathrm{tham}$ nhũng của các quốc gia. Kết quả này phù hợp với các nghiên cứu của Andersen (2009), Elbahnasawy (2014), Krishnan \& ctg (2013), Lio \& ctg (2011), Park \& ctg (2019) và Zhao \& ctg (2017).

Bên cạnh, kết quả nghiên cứu cũng cho thấy GDP bình quân đâu người (GDP), ổn định chính trị (PS) và chính phủ hiệu quả (GE) cũng góp phân đáng kể đến việc kiểm soát tham nhũng ở cấp độ quốc gia.

Cụ thể, tham số trung bình của biến GDP trong hai mô hình lần lượt có giá trị là 0,0018 và 0,0019 với xác suất ảnh hưởng tích cực của GDP đến biến CPI là $100 \%$, điều này cho thấy việc phát triển kinh tế là điều cần thiết để giúp kiểm soát tham nhũng và cung cấp ngân sách cho xây dựng và phát triển CPĐT. Kết quả này phù hợp với các nghiên cứu của Zhao \& ctg (2017) và Elbahnasawy (2014).

Tham số trung bình của biến PS lần lượt có giá trị là 1,4931 và 1,5394 với xác suất ảnh hưởng tích cực đến CPI là trên 96\%. Cuối cùng, tham số trung bình của biến GE lần lượt 5,8030 và 5,9202 với xác suất ảnh hưởng tích cực của GE đến CPI là $100 \%$. Kết quả này hàm ý rằng việc xây dựng một chính phủ ôn định và hiệu quả sẽ góp phân kiểm soát tốt tham nhũng. Những phát hiện này phù hợp với nghiên cứu của Basyal \& ctg (2018).

\section{2. Ảnh hưởng của chính phủ điện tử đến tham nhũng trong bối cảnh sự khác biệt văn hóa quốc gia}

Các Bảng 3 và Bảng 4 trình bày kết quả mô phỏng hậu nghiệm của Phương trình 2 bằng phương pháp hồi quy tuyến tính Bayes. Nhóm tác giả cũng tóm tắt những giá trị trung 


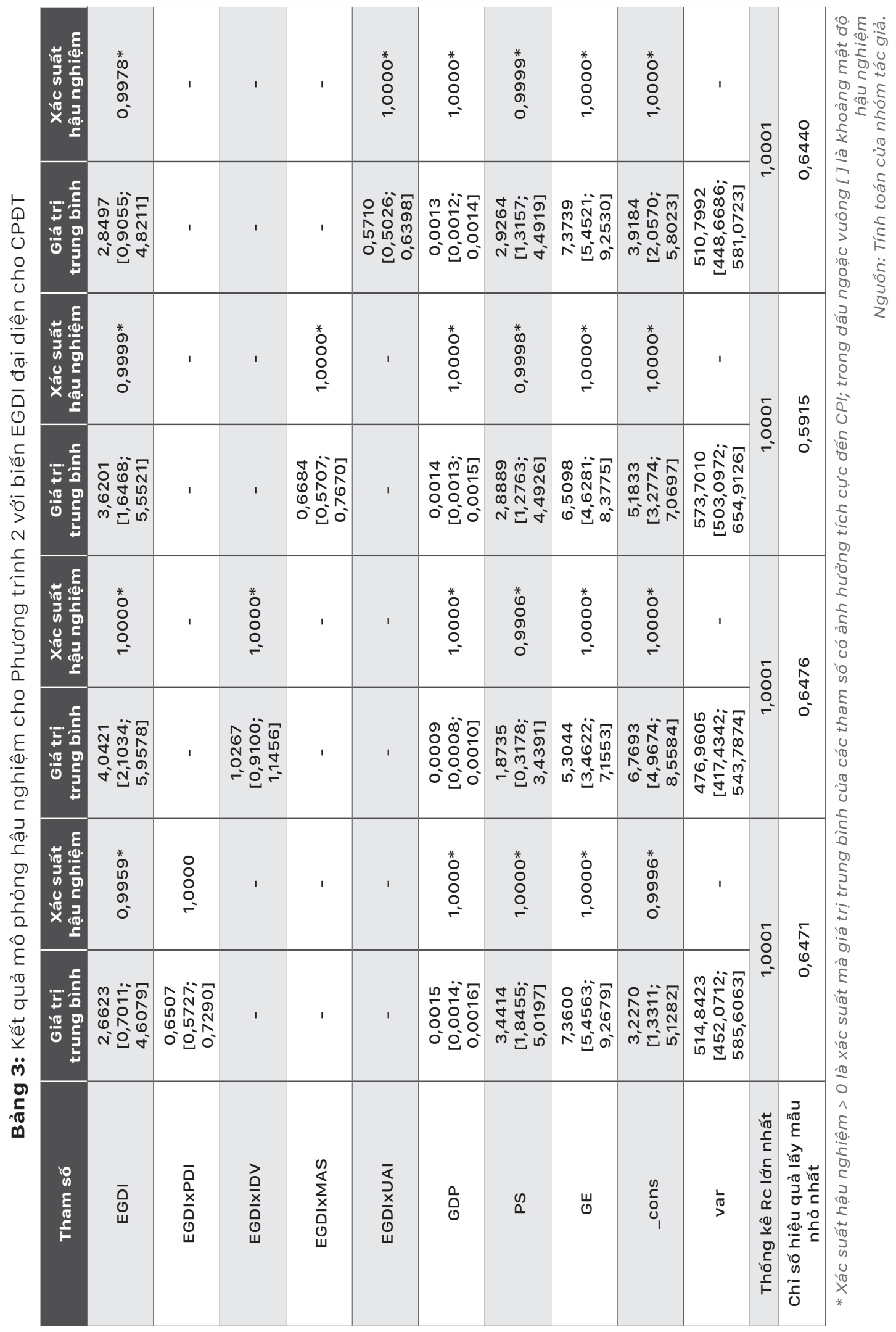




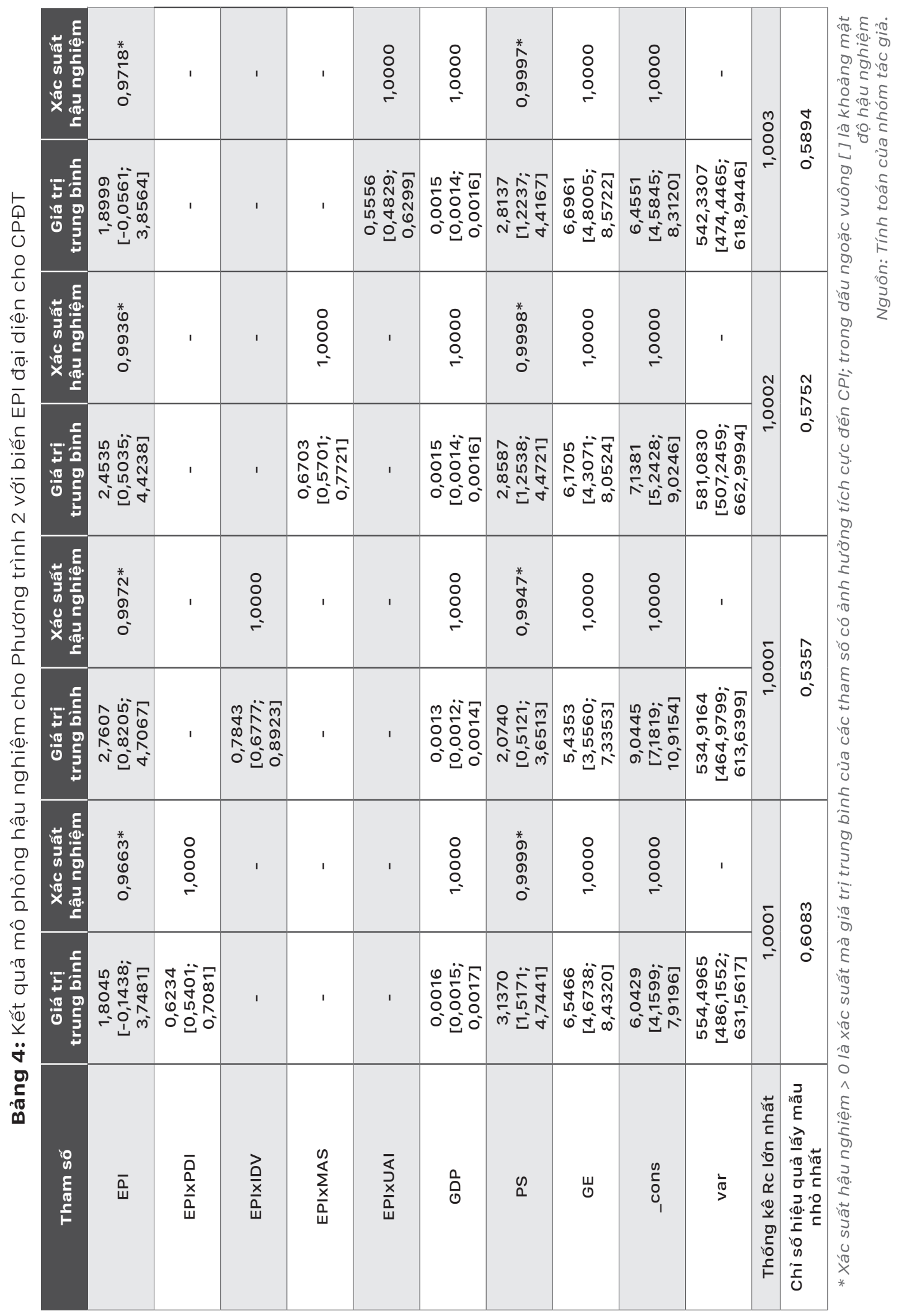


bình của các tham số mô hình thực, khoảng mật độ hậu nghiệm 95\% của phân phối hậu nghiệm và xác suất hậu nghiệm cũng được trình bày trong hai bảng này. Đồng thời, các Bảng 3 và Bảng 4 cũng cung cấp giá trị thống kê Rc và chỉ số lấy mẫu hiệu quả để đánh giá tính vững của suy luận Bayes.

Trường hợp biến đại diện cho CPĐT là EGDI. Kết quả Bảng 3 cho thấy, bốn giá trị thống kê Rc lớn nhất đều là 1,0001 (những giá trị Rc lớn nhất này đều nhỏ hơn 1,2 ). Vì vậy, MCMC đã đạt đến sự hội tụ. Bên cạnh đó, bốn chỉ số hiệu quả lấy mẫu nhỏ nhất của các tham số đêu lớn hơn 0,01 nên thuật toán MCMC đạt hiệu quả lấy mẫu (chỉ số hiệu quả lấy mẫu nhỏ nhất lần lượt là 0,$6471 ; 0,6476 ; 0,5915$ và $0,6440)$. Như vậy, kết quả chẩn đoán hội tụ và hiệu quả lấy mẫu đã cho thấy suy luận Bayes là vững.

Trong trường hợp biến đại diện cho CPĐT là EPI. Kết quả Bảng 4 cho thấy, bốn giá trị thống kê Rc lớn nhất lần lượt là 1,0001 ; 1,$0001 ; 1,0002$ và 1,0003 . Những giá trị thống kê lớn nhất đều nhỏ hơn 1,2 nên MCMC đã đạt đến sự hội tụ. Đồng thời, bốn chỉ số hiệu quả lấy mẫu nhỏ nhất của các tham số lần lượt là 0,$6083 ; 0,5357 ; 0,5752$ và 0,5894 . Tất cả chỉ số lấy mẫu hiệu quả đều lớn hơn 0,01 nên thuật toán MCMC đạt hiệu quả lấy mẫu. Như vậy, kết quả chẩn đoán hội tụ và hiệu quả lấy mẫu cũng cho thấy suy luận Bayes là vững.

Tại Bảng 3, kết quả mô phỏng hậu nghiệm cho thấy giá trị trung bình của biến EGDI lần lượt là 2,$6623 ; 4,0421 ; 3,6201$ và 2,8497 . Đồng thời, xác suất ảnh hưởng tích cực của biến EGDI lên biến CPI là trên $99 \%$. Bên cạnh đó, giá trị trung bình của các biến tương tác giữa EGDI lân lượt với các khía cạnh văn hóa (EGDIxPDI; EGDIxIDV; EGDIxMAS; EGDIxUAI) là 0,$6507 ; 1,0267 ; 0,6684$ và 0,5710 và xác suất ảnh hưởng tích cực của các biến tương tác này đến biến CPI là 100\%. Bảng 4 cung cấp kết quả mô phỏng hậu nghiệm trong trường hợp biến EPI đại diện cho CPĐT. Kết quả cho thấy giá trị trung bình của biến EPI dao động từ 1,8045 đến 2,7607 với xác suất ảnh hưởng tích cực của biến EPI đến biến CPI là trên $96 \%$. Các biến tương tác giữa $\mathrm{EPI}$ với các khía cạnh văn hóa (EPIxPDI; EPIxIDV; EPIxMAS; EPIxUAI) lần lượt là 0,6234 ; 0,$7843 ; 0,6703$ và 0,5556 . Đồng thời, xác suất ảnh hưởng tích cực của các biến tương tác này đêu là $100 \%$. Những kết quả này cho thấy sự khác biệt văn hóa có ảnh hưởng mạnh mẽ đến việc kiểm soát tham nhũng thông qua việc thực hiện CPĐT của các quốc gia. Do đó, Giả thuyết 2 được chấp nhận.

Thứ nhất, sự phát triển của CPĐT (EGDI) và tham gia $\mathrm{CPĐT}(\mathrm{EPI})$ của những quốc gia có nền văn hóa với khoảng cách quyền lực (PDI) càng lớn đã giúp kiểm soát tốt tham nhũng của các quốc gia này. Kết quả này cung cấp bằng chứng ngược lại so với các nghiên cứu trước (Nam, 2018; Zhao \& ctg, 2017).

Thư hai, kết quả nghiên cứu này cũng tìm thấy việc phát triển của CPĐT (EGDI) và tham gia $\mathrm{CPĐT} \mathrm{(EPI)} \mathrm{của} \mathrm{những} \mathrm{quốc} \mathrm{gia} \mathrm{có} \mathrm{nền}$ văn hóa với chủ nghĩa cá nhân (IDV) cao cũng có ảnh hưởng tích cực đến việc kiểm soát tham nhũng. Nghiên cứu của Nam (2018) cho thấy, nền văn hóa với chủ nghĩa cá nhân cao không có tác dụng chống tham nhũng của $\mathrm{CPĐT.}$

Thứ ba, trong một quốc gia có nền văn hóa định hướng nam quyên (MAS) càng cao thì sự phát triển của CPĐT (EGDI) và tham gia CPĐT (EPI) đã giúp kiểm soát tốt tham nhũng của các quốc gia này. Nam (2018) cũng cho thấy quốc gia với định hướng nam quyền cao sẽ giúp kiểm soát tham nhũng, tuy nhiên bằng chứng không có ý nghĩa thống kê.

Cuối cùng, kết quả cũng cho thấy việc phát triển CPĐT ở các nền văn hóa có sự né tránh rủi ro (UAI) cao đã giúp chính phủ kiểm soát tốt tham nhũng tại các quốc gia này. Kết quả này trái ngược với kết luận của Nam (2018) và Zhao \& ctg (2017).

Kết quả tại các Bảng 3 và Bảng 4 cũng cho thấy: (i) Tham số trung bình của yếu tố GDP 
bình quân đầu người (GDP) là dương và xác suất ảnh hưởng $100 \%$; (ii) Tham số trung bình của biến ổn định chính trị (PS) cũng có giá trị dương với xác suất ảnh hưởng trên $99 \%$; và (iii) Tham số trung bình của yếu tố chính phủ hiệu quả (GE) là dương và xác suất ảnh hưởng $100 \%$. Kết quả này cho thấy việc phát triển kinh tế kết hợp với ổn định chính trị và xây dựng chính hiệu quả là những yếu tố quan trọng để kiểm soát tham nhũng của các quốc gia.

\section{Kết luận và hàm ý chính sách}

Bài viết này xem xét ảnh hưởng của $\mathrm{CPĐT}$ đối với kiểm soát tham nhũng trong bối cảnh sự khác biệt văn hóa quốc gia. Bên cạnh đó, bài viết cũng phân tích ảnh hưởng của yếu tố kinh tế (GDP bình quân đầu người) và chính trị (ổn định chính trị và chính phủ hiệu quả) đến việc kiểm soát tham nhũng.

Kết quả phân tích bằng phương pháp hôi quy tuyến tính Bayes cho dũ liệu của 66 quốc gia trong giai đoạn 2003-2018 đã cho thấy, CPĐT được xem là một công cụ hiệu quả để kiểm soát tham nhũng. Bên cạnh đó, nghiên cứu cũng cho thấy sự khác biệt văn hóa quốc gia cũng có ảnh hưởng đến việc phát triển và tham gia CPĐT trong công cuộc chống tham nhũng. Cụ thể, các quốc gia với nền văn hóa có khoảng cách quyền lực lớn, chủ nghĩa cá nhân cao, định hướng nam quyền và né tránh rủi ro cao có tác động mạnh đến sự hiệu quả của $\mathrm{CPĐT}$ trong việc kiểm soát tham nhũng. Cuối cùng, nghiên cứu cũng thấy rằng GDP bình quân đầu người, ổn định chính trị và chính phủ hiệu quả là những yếu tố ảnh hưởng đến tham nhũng. Điều này ngụ ý rằng việc phát triển kinh tế, ổn định chính trị và chính phủ hiệu quả là điều cân thiết để giảm thiểu tham nhũng và đặc biệt phát triển kinh tế sẽ cung cấp ngân sách cho việc xây dựng và phát triên CPĐT.

Dựa trên kết quả nghiên cứu này, những nhà làm chính sách cân sử dụng sáng kiến $\mathrm{CPĐT} \mathrm{và} \mathrm{kết} \mathrm{hợp} \mathrm{với} \mathrm{các} \mathrm{công} \mathrm{cụ} \mathrm{chống} \mathrm{tham}$ nhũng khác (như phát triển kinh tế, ổn định chính trị và xây dựng chính phủ hiệu quả) trong việc giảm thiểu tham nhũng. Các sáng kiến CPĐT sẽ gia tăng sự minh bạch thông qua dũ liệu và thông tin mở, đông thời trao quyền cho người dân giám sát hoạt động của chính phủ. Bên cạnh đó, các nhà làm chính sách nên chú ý đến sự khác biệt trong văn hóa quốc gia. Một số quốc gia với những đặc điểm văn hóa được hưởng thành quả từ những nỗ lực chống tham nhũng do các sáng kiến thúc đẩy CPĐT, trong khi những quốc gia khác ở các nên văn hóa khác lại không giảm thiểu tham nhũng đáng kể. Vì vậy, các quy định toàn câu nói chung có thể có ít tác động đến giảm thiểu tham nhũng ở mỗi quốc gia hơn là áp lực từ khu vực.

Cuối cùng, bài viết này cũng có nhũ̃ng giới hạn nhất định. Thứ nhất, nghiên cứu chỉ tập trung xem xét ảnh hưởng của chính phủ điện tự đến tham nhũng trong bối cảnh sự khác biệt của bốn khía cạnh văn hóa của Hofstede. Do đó, các nghiên cứu tiếp theo cân bổ sung hai khía cạnh văn hóa còn lại của Hofstede, đó là định hướng dài hạn và tự thỏa mãn. Thứ hai, nghiên cứu chỉ tập trung vào phạm trù sự khác biệt văn hóa quốc gia trong mối quan hệ giữa $\mathrm{CPĐT} \mathrm{và} \mathrm{tham} \mathrm{nhũng.} \mathrm{Các} \mathrm{nghiên} \mathrm{cứu}$ trong tương lai cân bổ sung thêm các bối cảnh khác, chẳng hạn như tôn giáo. Cuối cùng, nghiên cứu này chỉ thực hiện một khám phá thực nghiệm ở cấp độ toàn câu của 66 quốc gia. Vì vậy, các nghiên cứu thực nghiệm trong tương lai cần phân tích tham nhũng ở cấp độ quốc gia hoặc khu vực kết hợp với sự khác biệt trong bối cảnh chính trị. 


\section{Tài liệu tham khảo}

Andersen, T. B. (2009). E-Government as an anti-corruption strategy. Information Economics and Policy, 21(3), 201-210.

Basyal, D. K., Poudyal, N., \& Seo, J. W. (2018). Does E-government reduce corruption? Evidence from a heterogeneous panel data model. Transforming Government: People, Process and Policy, 12(2), 134-154.

Berrell, M. (2021). National Culture and the Social Relations of Anywhere Working. In Anywhere Working and the Future of Work (pp. 23-59). Hershey, Pennsylvania: IGI Global.

Brooks, S. P., \& Gelman, A. (1998). General Methods for Monitoring Convergence of Iterative Simulations. Journal of Computational and Graphical Statistics, 7(4), 434-455.

Elbahnasawy, N. G. (2014). E-Government, Internet Adoption, and Corruption: An Empirical Investigation. World Development, 57, 114-126.

Ha, C. N. (2020). Posterior Summary of Bayes Error Using Monte-Carlo Sampling and Its Application in Credit Scoring, Asian Journal of Economics and Banking, 4(2),117-126.

Hofstede, G. (1984). Culture's Consequences: International Differences in Work-Related Values. Beverly Hills: SAGE Publications.

House, R. J., Hanges, P. J., Javidan, M., Dorfman, P. W., \& Gupta, V. (2004). Culture, leadership, and organizations: The GLOBE study of 62 societies. Beverly Hills: Sage publications.

Jaeger, P. T. (2006). Assessing Section 508 compliance on federal e-government Web sites: A multi-method, user-centered evaluation of accessibility for persons with disabilities. Government Information Quarterly, 23(2), 169-190.

Jain, A. K. (2001). Corruption: A Review. Journal of Economic Surveys, 15(1), 71-121.

Klitgaard, R. (1988). Controlling Corruption. Berkeley: University of California Press.

Krishnan, S., Teo, T. S. H., \& Lim, V. K. G. (2013). Examining the relationships among e-government maturity, corruption, economic prosperity and environmental degradation: A cross-country analysis. Information \& Management, 50(8), 638-649.

Kruschke, J. K., Aguinis, H., \& Joo, H. (2014). The time has come: Bayesian methods for data analysis in the organizational sciences: Erratum. Organizational Research Methods, 17(1), 107-107.

Linh, N. T. X. (2020). Social Existence Determines Consciousness: How the Economy Matters for Cultural Changes? A Study of Selected Asian Countries. Asian Journal of Economics and Banking, 4(1), 127-146.

Lio, M. C., Liu, M. C., \& Ou, Y. P. (2011). Can the internet reduce corruption? A cross-country study based on dynamic panel data models. Government Information Quarterly, 28(1), 47-53.

Manoharan, A. (2013). A Three Dimensional Assessment of U.S. County e-Government. State and Local Government Review, 45(3), 153-162.

Nam, T. (2018). Examining the anti-corruption effect of e-government and the moderating effect of national culture: A cross-country study. Government Information Quarterly, 35(2), 273-282. 
Nguyen, D. V., \& Duong, M. T. H. (2021). Shadow Economy, Corruption and Economic Growth: An Analysis of BRICS Countries. The Journal of Asian Finance, Economics and Business, 8(4), 665-672.

Nguyen, V. D., \& Duong, T. H. M. (2022). Corruption, Shadow Economy, FDI, and Tax Revenue in BRICS: A Bayesian Approach. Montenegrin Journal of Economics, 18(2), 55-64.

Oanh, T. T. K., Diep, N. V., Truyen, P. T., \& Chau, N. X. B. (2022). The impact of public expenditure on economic growth of provinces and cities in the Southern Key Economic Zone of Vietnam: Bayesian approach. In N. Ngoc Thach, D. T. Ha, N. D. Trung, \& V. Kreinovich (Eds.), Prediction and Causality in Econometrics and Related Topics (pp. 328-344). Cham: Springer International Publishing.

Park, C. H., \& Kim, K. (2019). E-government as an anti-corruption tool: panel data analysis across countries. International Review of Administrative Sciences, 86(4), 691-707.

Roberts, G. O., \& Rosenthal, J. S. (2001). Optimal scaling for various Metropolis-Hastings algorithms. Statistical Science, 16(4), 351-367.

Rose-Ackerman, S. (1996). The Political Economy of Corruption: Causes and Consequences. Viewpoint, 74, 1-4.

Seligson, M. A. (2002). The Impact of Corruption on Regime Legitimacy: A Comparative Study of Four Latin American Countries. The Journal of Politics, 64(2), 408-433.

Thach, N. N. (2021). How Values Influence Economic Progress? Evidence from South and Southeast Asian Countries. In N. Ngoc Thach, V. Kreinovich, \& N. D. Trung (Eds.), Data Science for Financial Econometrics (pp. 207-221). Cham: Springer International Publishing.

Thach, N. N., Anh, L. H., \& An, P. T. H. (2019). The effects of public expenditure on economic growth in Asia countries: A Bayesian model averaging approach. Asian Journal of Economics and Banking, 3(1), 126-149.

Trafimow, D., Amrhein, V., Areshenkoff, C. N., Barrera-Causil, C. J., Beh, E. J., Bilgiç, Y. K., ... Cepeda-Freyre, H. A. (2018). Manipulating the alpha level cannot cure significance testing. Frontiers in Psychology, 9, 699.

Villoria, M., Van Ryzin, G. G., \& Lavena, C. F. (2013). Social and Political Consequences of Administrative Corruption: A Study of Public Perceptions in Spain. Public Administration Review, 73(1), 85-94.

Vladik, K., Olga, K., Nguyen, N. T., \& Nguyen, D. T. (2019). Use of Symmetries in Economics: An Overview. Asian Journal of Economics and Banking, 3(1), 20-39.

Wasserstein, R. L., Schirm, A. L., \& Lazar, N. A. (2019). Moving to a World Beyond "p < 0.05". The American Statistician, 73(sup1), 1-19.

Zhao, H., Ahn, M. J., \& Manoharan, A. P. (2017). E-government, corruption reduction and culture: a study based on panel data of 57 countries. Paper presented at the Proceedings of the 18th Annual International Conference on Digital Government Research, Staten Island, NY, USA. 


\title{
E-Government, National Culture and Corruption: Evidence from Bayesian Approach
}

\author{
Nguyen Van Diep ${ }^{(*)}$, \\ Pham Xuan Thu, Nguyen Hoang Thi
}

Received: 21 May 2021 | Revised: 02 August 2021 | Accepted: 10 August 2021

\begin{abstract}
This paper aims to analyze the influence of e-government on corruption in the context of cultural differences between countries. The Bayesian linear regression method is used to process panel datasets of 66 countries from 2003 to 2018 . The results show a strong and positive influence of e-government on the control of corruption. In addition, the extent to which e-government influences corruption control depends on the national cultural context. In particular, the development and participation of e-government will help control corruption more effectively in countries with cultures where the degree of power distance, individualism, masculinity, and uncertainty avoidance high. Also, the research results show that GDP per capita, political stability, and government effectiveness have a positive effect on controlling corruption. This result implies that, besides cultural factors, economic and political factors also reduce corruption at the national level.
\end{abstract}

KEYWORDS: Bayesian Approach, Corruption, Culture, E-government.

JEL classification: C11, D73, H11, Z10.

\section{Nguyen Van Diep}

Email: diep.nv@ou.edu.vn.

${ }^{(*)}$ Ho Chi Minh City Open University;

35-37 Ho Hao Hon Street, Co Giang Ward, District 1, Ho Chi Minh City. 